\title{
NRSF/REST levels are decreased in cholangiocellular carcinoma but not hepatocellular carcinoma compared with normal liver tissues: A tissue microarray study
}

\author{
YANLAN YU ${ }^{1 *}$, SHAN LI $^{1 *}$, HUIYAN ZHANG ${ }^{2}$, XUQING ZHANG ${ }^{2}$, DEYU GUO ${ }^{3}$ and JIQIANG ZHANG ${ }^{1}$ \\ ${ }^{1}$ Department of Neurobiology, Chongqing Key Laboratory of Neurobiology, Third Military Medical University; Departments \\ of ${ }^{2}$ Infectious Diseases and ${ }^{3}$ Pathology, Southwest Hospital, Third Military Medical University, Chongqing 400038, P.R. China
}

Received July 9, 2016; Accepted September 15, 2017

DOI: $10.3892 / \mathrm{ol} .2018 .8169$

\begin{abstract}
The transcription factor neuron-restrictive silencer factor (NRSF), also termed repressor element 1-silencing transcription factor (REST), has been previously demonstrated to repress the expression of neuronal genes in non-neuronal cells, facilitating the controlled development and organization of nerve tissue. However, previous studies have reported NRSF/REST to be upregulated or downregulated in multiple types of carcinoma. Liver diseases are a major global health concern, with cirrhosis and liver carcinoma among the most common causes of mortality worldwide. A previous study demonstrated that there were $>400 \mathrm{NRSF} / \mathrm{REST}$ target genes in mouse liver cells; however, the expression profile of NRSF/REST in human liver disease remains unclear. The present study examined NRSF/REST expression in human normal and liver carcinoma samples using tissue microarray immunohistochemistry. The results demonstrated that in normal liver tissues, NRSF/REST can be detected in the cytoplasm and nuclei of the cell; whereas in the liver carcinoma tissue, NRSF/REST is only detected in the cytoplasm. Furthermore, the number of samples with high levels of NRSF/REST was significantly lower in cholangiocellular carcinoma samples compared with normal tissues. Additionally, no detectable sex- or age-associated differences were identified
\end{abstract}

Correspondence to: Dr Jiqiang Zhang, Department of Neurobiology, Chongqing Key Laboratory of Neurobiology, Third Military Medical University, 30 Gaotanyan Street, Shapingba, Chongqing 400038, P.R. China

E-mail: zhangjqtmmu@yahoo.com

Dr Deyu Guo, Department of Pathology, Southwest Hospital, Third Military Medical University, 30 Gaotanyan Street, Shapingba, Chongqing 400038, P.R. China

E-mail: gdy007@tmmu.edu.cn

*Contributed equally

Key words: neuron-restrictive silencer factor, repressor element 1-silencing transcription factor, liver carcinoma, tissue microarray, immunohistochemistry in NRSF/REST expression among all the tissues examined. In conclusion, the results of the present study revealed nuclear loss of NRSF/REST in hepatic carcinomas and decreased expression of NRSF/REST in cholangiocellular carcinoma, indicating that the cytoplasmic translocation of NRSF/REST may be involved in liver tumorigenesis. A low expression level of NRSF/REST may be a novel biomarker for cholangiocellular carcinoma.

\section{Introduction}

Neuron-restrictive silencer factor (NRSF), also termed repressor element 1-silencing transcription factor (REST), is a zinc-finger transcription factor and an important regulator of neural genes (1). Chong et al (2) first reported that NRSF/REST is a silencer protein that reduces the expression of sodium channel genes in neurons. A previous study demonstrated that $\mathrm{NRSF} / \mathrm{REST}$ is an important regulator of neurogenesis in vitro and in vivo. For example, downregulation of NRSF/REST in embryonic stem cells induces neuronal lineage differentiation (3), and knockdown of NRSF/REST in cultured neural stem cells induces the expression of pro-neuronal genes, including neuronal differentiation 1 , neuron-specific class III $\beta$-tubulin and doublecortin (4). In Xenopus and chicken embryos, NRSF/REST inactivation induces abnormal neurogenesis and inhibits the repression of neuronal tubulin and several other neuronal target genes $(5,6)$. Additionally, NRSF/REST overexpression represses the expression of neuronal genes, including $\mathrm{N}$-tubulin and neuronal cell adhesion molecule (7).

Numerous studies have investigated the expression and, to a lesser extent, the function of NRSF/REST in tumors of the nervous system (8-15). Certain tumors, including neuroblastoma, share a number of biological properties with neuronal progenitor cells and, thus, can acquire neuronal phenotypes in response to a variety of agents. A study by Nishimura et al (8) examined the levels of NRSF/REST mRNA in a human neuroblastoma cell line following induced differentiation. The study demonstrated that the NRSF/REST mRNA level was evidently decreased following induction, indicating that NRSF/REST expression is a biochemical marker of neuronal differentiation in neuroblastoma cells (8). A similar downregulation of NRSF/REST was also demonstrated in other studies using different neuroblastoma cell lines $(9,10)$. In addition, 
high NRSF/REST levels have been detected in human medulloblastoma cell lines and tumors (11-13). Similarly, in human glioblastoma multiforme (GBM), NRSF/REST is highly expressed (14) and its inhibition suppresses the proliferation and migration of GBM cells (15).

Certain studies have also investigated the expression and/or function of NRSF/REST in non-neuroepithelial tumors in vitro and in vivo. Gurrola-Diaz et al (16) reported that exogenous overexpression of NRSF/REST in NRSF/REST-deficient small-cell lung cancer (SCLC) cell lines induced apoptosis of SCLC cells, indicating that the inhibition of NRSF/REST activity is a crucial step in the carcinogenesis of a subgroup of SCLCs (16). Similar results were also observed in human non-SCLC cell lines (17). Kreisler et al (18) reported that loss of NRSF/REST expression was associated with the malignant progression of SCLC. In human breast cancer cells, NRSF/REST activity was required for estradiol stimulation of the cell cycle (19). Immunohistochemistry previously demonstrated that NRSF/REST expression is significantly lower in breast cancer samples compared with normal and benign breast samples, and that knockdown of NRSF/REST expression by short hairpin RNA in MCF-7 human breast cancer cells resulted in an increase in cell proliferation, suppression of apoptosis and reduced sensitivity to anticancer drugs (20). Previous studies have also demonstrated the important function of NRSF/REST in the pathogenesis of uterine fibroids (21). However, the expression of NRSF/REST in liver tumors remains unclear. Thus, the present study determined the expression profile of NRSF/REST in liver tumors using tissue microarray (TMA) immunohistochemistry.

\section{Materials and methods}

TMA and pathology. All paraffin-embedded TMAs used in the present study were purchased from US Biomax, Inc. (Thermo Fisher Scientific, Inc., Waltham, MA, USA). The hepatic carcinoma and normal hepatic tissue TMAs (cat. no. BC03118) contained 90 carcinoma samples, including 15 cholangiocellular carcinoma (CCC), 75 hepatocellular carcinoma (HCC), and 10 normal hepatic tissue samples. On the basis of morphology, the liver carcinoma samples were graded 1-3 (or I-III), according to the Tumor-Node-Metastasis grading system (22) by the supplier, indicating well-, moderately- or poorly-differentiated tissue, respectively. In total, there were 200 tissue samples on the microarray, with two samples from each patient.

Immunohistochemistry. TMAs were deparaffinized with xylene, rehydrated with a graded alcohol series and subjected to heat-mediated antigen retrieval [0.01 M sodium citrate buffer, (pH 6.0)], according to a previously described protocol $(23,24)$. TMAs were then rinsed with phosphate-buffered saline [PBS; $0.01 \mathrm{~mol} / \mathrm{l},(\mathrm{pH} 7.4)]$ and blocked with $3 \% \mathrm{H}_{2} \mathrm{O}_{2}(\mathrm{v} / \mathrm{v}$ in PBS) for $15 \mathrm{~min}$ at room temperature. The sections were then incubated for $20 \mathrm{~min}$ at room temperature with $2 \%$ normal goat serum (v/v; Beijing Zhongshan Golden Bridge Biotechnology Co., Ltd., Beijing, China) at room temperature to block the non-specific binding. Subsequently, the TMAs were incubated overnight at $4^{\circ} \mathrm{C}$ with a polyclonal rabbit antibody against NRSF/REST (cat. no. ab21635; Abcam,
Cambridge, UK) that was diluted 1:100 with antibody diluent (cat. no. S3022; Dako; Agilent Technologies, Inc., Santa Clara, CA, USA). Following several washes with PBS, the sections were incubated with biotinylated goat anti-rabbit secondary antibody (cat. no. ZB2010; 1:200; Beijing Zhongshan, China) for $1 \mathrm{~h}$ at room temperature. The sections were then washed with PBS and incubated with horseradish peroxidase-labeled streptavidin (cat. no. ZB2404; Beijing Zhongshan Golden Bridge Biotechnology Co., Ltd.) for $1 \mathrm{~h}$ at room temperature. Finally, the sections were incubated with a diaminobenzidine-peroxidase substrate kit (cat. no. ZLI-9018; Beijing Zhongshan Golden Bridge Biotechnology Co., Ltd.) for 5 min at room temperature. The equivalent procedure was conducted for the blank controls, with the primary antibody replaced by antibody diluent.

Imaging and data analysis. Images of the immunohistochemical staining were captured with a DP70 digital camera (Leica Microsystems GmbH, Wetzlar, Germany) mounted to a BX60 Olympus microscope (Olympus Corporation, Tokyo, Japan). The staining was scored according to the previously described four-point system (score 0-3) (24) by a pathologist (double-blinded) as follows: Score 3, dark staining that is easily visible and present in $>50 \%$ of cells; score 2 , focal areas of dark staining $(<50 \%$ of cells) or moderate staining of $>50 \%$ of cells; score 1 , focal moderate staining in $<50 \%$ of cells or pale staining in any proportion of cells not easily observable at low power; and score 0 , none of the above. A high level of expression was defined as a score of 2-3 and low level of expression was defined as a score of $0-1$, as described previously (24). Considering the comparatively small sample size, an early tumor stage was defined as stages I and II, and the advanced stage was defined as stages III and IIIb. Well-differentiated carcinoma (WDC) was defined as grade 1, moderately-differentiated carcinoma (MDC) as grade 2 and poorly-differentiated carcinoma (PDC) was defined as grade 3 (24).

Statistical analysis. All data are expressed as n (\%) and were compared using a $\chi^{2}$ test. A Fisher's exact test was used for correction when necessary. Statistical analysis was performed using SPSS software (version 18.0; SPSS, Inc., Chicago, IL, USA). All P-values were 2-tailed and $\mathrm{P}<0.05$ was considered to indicate a statistically significant difference.

\section{Results}

Subcellular localization of NRSF/REST immunohistochemical staining. The immunohistochemical analysis demonstrated that, in normal hepatic tissue, NRSF/REST was present in the nuclei and cytoplasm of hepatocytes and cholangiocytes (Fig. 1A and B). In CCC and HCC tissues, NRSF/REST was predominantly detected in the cytoplasm, with the nuclei clearly unstained. Additionally, the NRSF/REST immunohistochemical staining of HCC tissues seemed stronger than that of CCC tissues (Fig. 1C-F).

Expression of NRSF/REST in liver carcinoma. The levels of NRSF/REST immunoreactivity were compared between normal and liver carcinomas. As presented in Table I, among the 10 cases of normal liver tissue on the TMA, $90 \%$ exhibited 


\section{Normal liver}
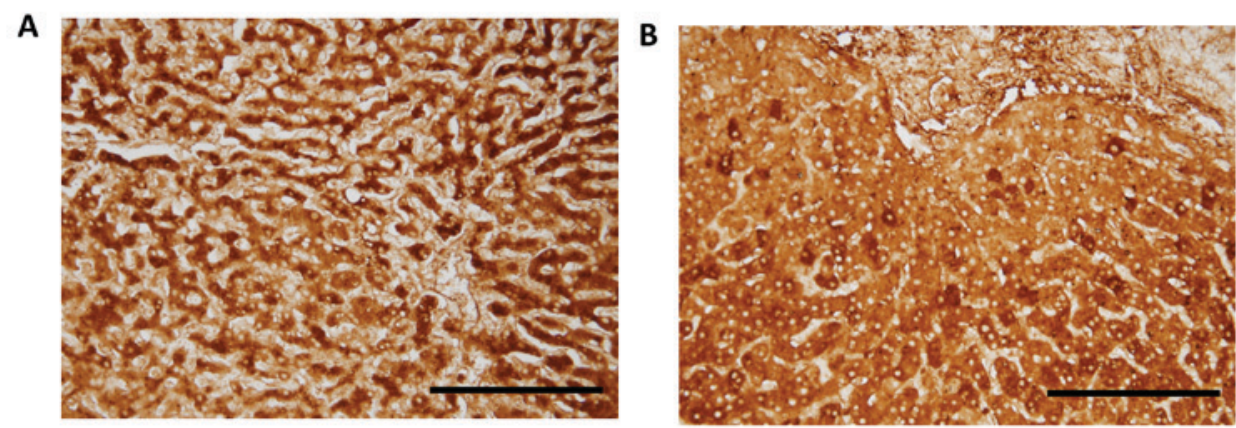

HCC
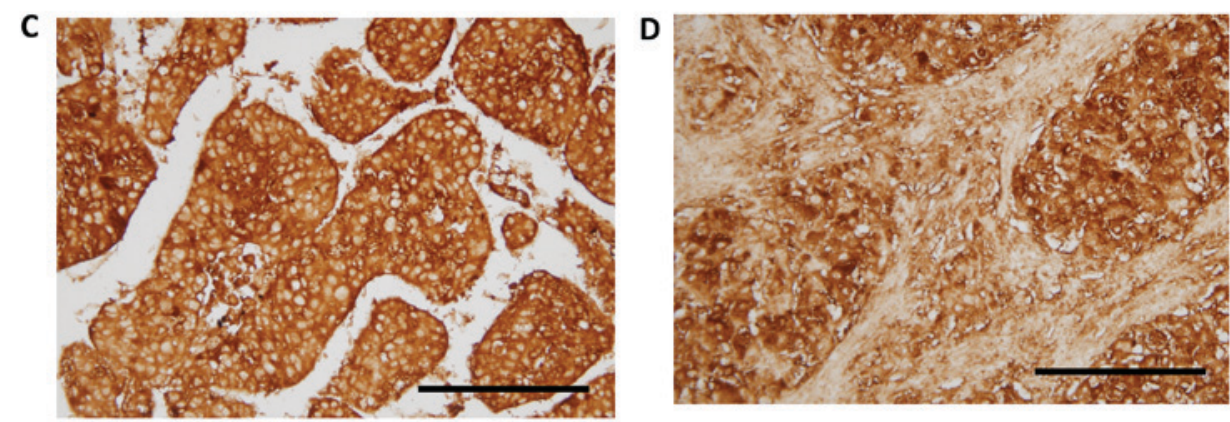

\section{CCC}
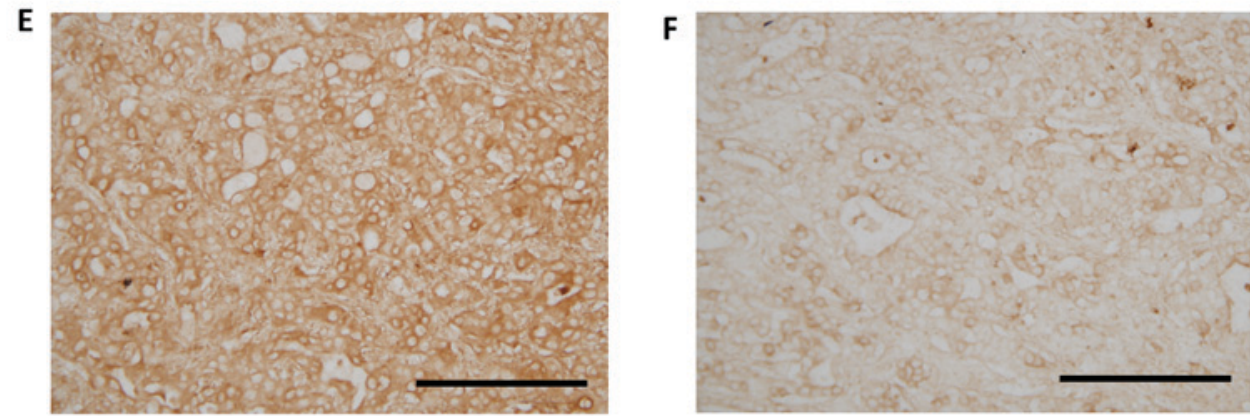

Figure 1. Representative immunohistochemical staining for NRSF/REST in normal and cancerous liver tissues. Normal liver tissue from (A) a 40-year-old female and (B) a 3-year-old male. HCC tissue from (C) a 45-year-old female (grade 2; stage III) and (D) a 51 year-old male (grade 2; stage III). CCC tissue from (E) a 24-year-old male (grade 2; stage III) and (F) a 40-year-old female (grade 1; stage III). Scale bar, $200 \mu \mathrm{m}$. The number of samples with high levels of NRSF/REST was increased in normal liver tissue and HCC as compared with CCC. Additionally, in normal liver tissue, nuclear and cytoplasmic NRSF/REST expression was detected; whereas in liver carcinomas, NRSF/REST was predominantly detected in the cytoplasm. NRSF/REST, neuron-restrictive silencer factor/repressor element 1-silencing transcription factor; HCC, hepatocellular carcinoma; CCC, cholangiocellular carcinoma.

high levels of NRSF/REST (score 2-3; Fig. 1A and B). Among the 90 cases, 66\% (59/90) of liver carcinoma samples and $77 \%$ (58/75) of HCC samples demonstrated high levels of NRSF/REST (Table I; Fig. 2). There was no significant difference in the percentage of samples with high NRSF/REST levels between the normal liver tissue and liver carcinoma samples ( $\mathrm{P}>0.05$; Fig. 2A; Table I) or between normal liver tissue and HCC (P>0.05; Fig. 2B; Table I). However, the percentage of samples with high NRSF/REST expression was significantly reduced in CCC samples $(7 \% ; 1 / 15)$ compared with normal liver tissues ( $\mathrm{P}<0.001$; Fig. $2 \mathrm{C}$; Table I) and with HCC samples ( $\mathrm{P}<0.001$; Fig. 2D; Table I).

Among the 90 cases of liver carcinomas, 11 cases were graded as WDC, 64 as MDC and 11 as PDC (including grade 2-3). The grading of the remaining 4 cases was undetermined. The percentage of high NRSF/REST immunoreactivity was 73\% (8/11) in WDCs, 69\% (44/64) in MDCs and 36\% (4/11) in PDCs. There was no statistical difference between the percentages of samples with high NRSF/REST staining among the three groups ( $\mathrm{P}>0.05$; Fig. 2E; Table I).

Additionally, the association between NRSF/REST expression and tumor stage was analyzed. Stages I and II were defined as early stage, and stages III and IIIb as advanced stage. Thus, 43 cases were early stage and 47 cases were advanced stage. High levels of NRSF/REST were observed in $67 \%$ (29/43) of early stage and 64\% (30/47) of advanced stage samples, with no statistical difference between the two stages (P>0.05; Fig. 2F; Table I).

Sex-associated differences in NRSF/REST in normal and cancerous liver tissue. The occurrence of liver diseases exhibits a certain degree of sex bias, with a higher percentage 
Table I. Expression of NRSF/REST in normal and abnormal liver tissues.

\begin{tabular}{lrrrr}
\hline Tissue & High & Low & $\chi^{2}$ & P-value \\
\hline Carcinoma vs. normal & & & 1.476 & 0.224 \\
$\quad$ Carcinoma & 59 & 31 & & \\
$\quad$ Normal & 9 & 1 & & \\
CCC vs. normal & & & 14.063 & $<0.001$ \\
CCC & 1 & 14 & & \\
Normal & 9 & 1 & & \\
HCC vs. normal & & & 0.259 & 0.611 \\
HCC & 58 & 17 & & \\
Normal & 9 & 1 & & \\
CCC vs. HCC & & & 27.645 & $<0.001$ \\
CCC & 1 & 14 & & \\
HCC & 58 & 17 & & \\
Carcinoma early/ & & & 0.130 & 0.719 \\
advanced stage & & & & \\
Early & 29 & 14 & & \\
Advanced & 30 & 17 & & \\
Carcinoma & & & 4.656 & \\
differentiation stage & & & & \\
WDC & & & & \\
MDC & 4 & 20 & & \\
PDC & & & & \\
\hline
\end{tabular}

High NRSF/REST expression was defined as score 2-3 and low expression as score $0-1$. WDC was defined as grade 1 , MDC was defined as grade 2 and PDC was defined as grade 3 and grade 2-3. Early stage was defined as stage I-II and advanced stage was defined as stage III (including IIIb). NRSF/REST, neuron-restrictive silencer factor/repressor element 1-silencing transcription factor; HCC, hepatocellular carcinoma; CCC, cholangiocellular carcinoma; WDC, well-differentiated carcinoma; MDC, moderately-differentiated carcinoma; PDC, poorly-differentiated carcinoma.

detected in males. A total of $74.11 \%$ of new liver cancer cases in 2014 in the USA were in males (25). Thus, the present study compared the expression of NRSF/REST in normal and cancerous liver tissues from men and women. Among the 10 cases of normal liver tissue, the percentage of samples with high NRSF/REST levels was $83 \%(5 / 6)$ in males and $100 \%$ (4/4) in females, with no significant difference between the sexes $(\mathrm{P}>0.05$; Fig. 3A; Table II). Among the 90 cases of liver carcinoma, the percentage of samples with high levels of NRSF/REST was $70 \%(51 / 73)$ in males and $47 \%(8 / 17)$ in females, demonstrating no significant difference between the sexes ( $\mathrm{P}>0.05$; Fig. 3B; Table II).

Among the 15 cases of CCC, only 1 male demonstrated high levels of NRSF/REST and the other 14 cases ( 7 male and 7 female) all demonstrated low levels of NRSF; thus, no significant sex-associated difference was identified ( $\mathrm{P}>0.05$; Table II). Among the 75 cases of HCC, the percentage of samples with high levels of NRSF/REST was $77 \%(50 / 65)$ in males and $80 \%(8 / 10)$ in females; thus, there was no significant sex-associated difference detected $(\mathrm{P}>0.05$; Table II).
Table II. Sex and age-associated differences of NRSF/REST in normal and cancerous liver tissues.

\begin{tabular}{lcccc}
\hline \multicolumn{3}{l}{ A, Sex-associated differences } & & \\
\hline Tissue & High & Low & $\chi^{2}$ & P-value \\
\hline Normal & & & 0.000 & 1.000 \\
Male & 5 & 1 & & \\
Female & 4 & 0 & & 0.075 \\
Carcinoma & & & 3.176 & \\
Male & 51 & 22 & & 1.000 \\
Female & 8 & 9 & & \\
CCC & & & 0.000 & \\
Male & 1 & 7 & & \\
Female & 0 & 7 & & \\
HCC & & & 0.000 & \\
Male & 50 & 15 & & \\
Female & 8 & 2 & & \\
\hline
\end{tabular}

B, Age-associated differences

\begin{tabular}{lcccc}
\hline Tissue & High & Low & $\chi^{2}$ & P-value \\
\hline $\begin{array}{l}\text { Normal } \\
\geq 26.8\end{array}$ & 3 & 1 & 0.046 & 0.830 \\
$<26.8$ & 6 & 0 & & \\
Carcinoma & & & 0.945 & 0.331 \\
$\geq 50.4$ & 26 & 17 & & \\
$<50.4$ & 33 & 14 & & \\
CCC & & & 0.005 & 0.945 \\
$\geq 48.5$ & 0 & 8 & & \\
$<48.5$ & 1 & 6 & & 0.373 \\
HCC & & & 0.792 & \\
$\geq 50.8$ & 27 & 10 & & \\
$<50.8$ & 31 & 7 & & \\
\hline
\end{tabular}

High levels of NRSF/REST expression were defined as score 2-3 and low levels of expression were defined as score 0-1. Ages are presented in years. No sex- or age-associated differences were detected. NRSF/REST, neuron-restrictive silencer factor/repressor element 1-silencing transcription factor; HCC, hepatocellular carcinoma; CCC, cholangiocellular carcinoma.

Age-associated differences in NRSF/REST in normal and cancerous liver tissue. The present study also investigated the effect of age on NRSF/REST immunoreactivity in normal and cancerous liver tissues. Among the 10 normal liver tissues, the mean age of the patients was 26.8 years. The percentage of samples with high expression of NRSF/REST was $75 \%(3 / 4)$ in samples from patients aged $\geq 26.8$ years, and $100 \%(6 / 6)$ in those aged $<26.8$ years. The statistical analysis demonstrated no significant difference between the two age groups $(\mathrm{P}>0.05$; Fig. 3C; Table II). Among the 90 cases of liver carcinoma, the mean age was 50.4 years. The percentage of samples with high NRSF/REST expression was $60 \%(26 / 43)$ in patients 
A

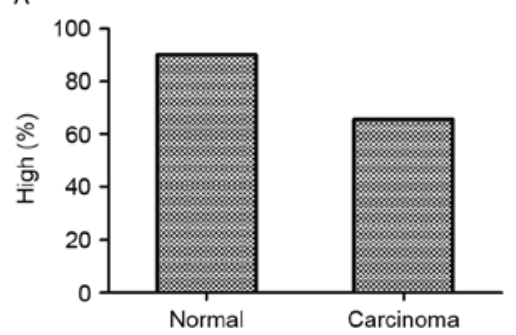

D

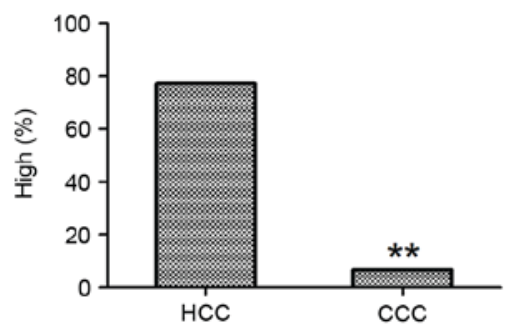

B
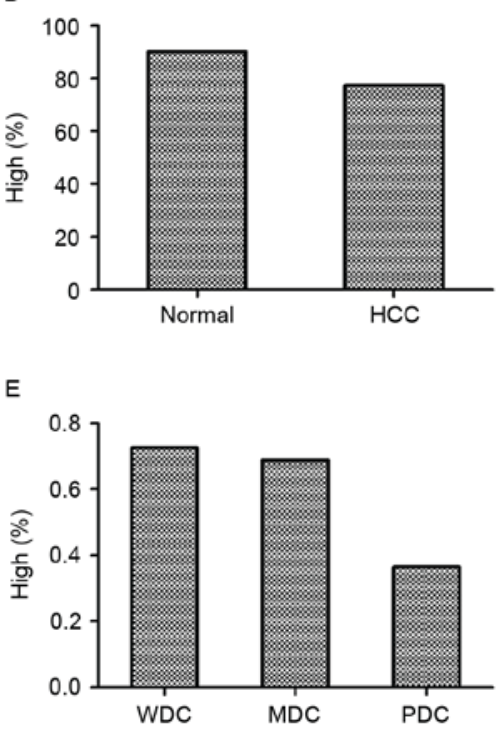

C
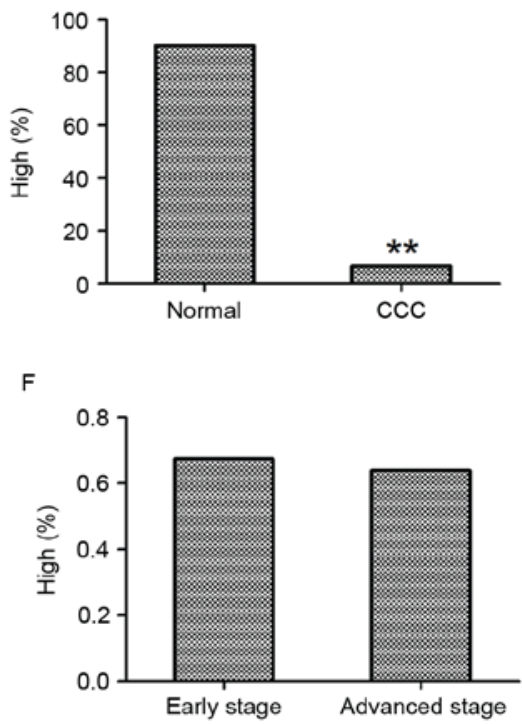

Figure 2. Statistical analysis of NRSF/REST immunoreactivity in normal and cancerous liver tissues and the association with tumor differentiation and stage. (A) Percentage of samples with high NRSF/REST levels between normal and liver carcinoma tissues. (B) Percentage of samples with high NRSF/REST levels between normal and HCC liver tissues. (C) Percentage of samples with high NRSF/REST levels between CCC and normal liver tissue. (D) Percentage of samples with high NRSF/REST levels between CCC and HCC. (E) Percentage of samples with high NRSF/REST levels among the differentiation status. (F) Percentage of samples with high NRSF/REST levels between tumor stages. ${ }^{* *} \mathrm{P}<0.001$. NRSF/REST, neuron-restrictive silencer factor/repressor element 1-silencing transcription factor; HCC, hepatocellular carcinoma; CCC, cholangiocellular carcinoma; WDC, well-differentiated carcinoma; MDC, moderately-differentiated carcinoma; PDC, poorly-differentiated carcinoma.

A

Normal

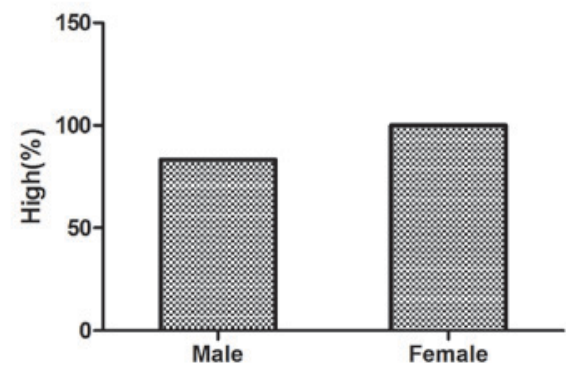

C

Normal

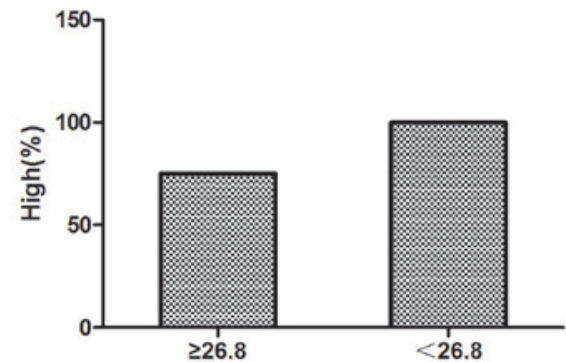

B

Carcinoma

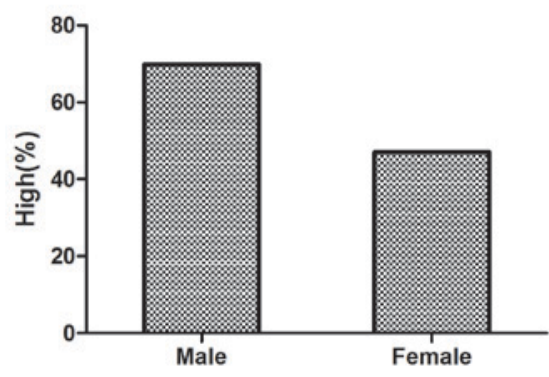

Carcinoma

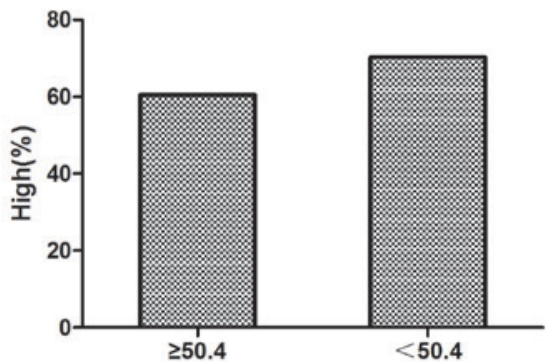

Figure 3. Sex and age analysis of NRSF/REST immunoreactivity in normal and cancerous liver tissues. Sex-associated differences of NRSF/REST levels in (A) normal liver tissue and (B) hepatic carcinoma. Age-associated differences of NRSF/REST levels in (C) normal liver tissue and (D) hepatic carcinoma. No sex- or age-associated differences were detected among the liver tissues. NRSF/REST, neuron-restrictive silencer factor/repressor element 1-silencing transcription factor.

aged $\geq 50.4$ years and $70 \%(33 / 47)$ in those aged $<50.4$ years Statistical analysis demonstrated no significant difference between the two age groups ( $\mathrm{P}>0.05$; Fig. 3D; Table II).
The age-associated difference of NRSF/REST immunoreactivity in individual liver carcinoma tissues was also analyzed. Among the 15 cases of CCC, the mean age of the patients was 
48.5 years. Only 1 patient aged $<48.5$ years demonstrated high levels of NRSF/REST and statistical analysis revealed no significant difference between the two age groups $(\mathrm{P}>0.05$; Table II). Among the 75 cases of HCC, the mean age of the patients was 50.8 years. The percentage with high expression of NRSF/REST was $73 \%(27 / 37)$ in patients $\geq 50.8$ years old and $82 \%(31 / 38)$ in those $<50.8$ years old. Statistical analysis demonstrated no significant difference between the two age groups ( $\mathrm{P}>0.05$; Table II).

\section{Discussion}

The present study examined the expression of NRSF/REST in normal liver tissue and liver carcinomas using TMA immunohistochemistry. The results demonstrated that, in the normal liver tissue, NRSF/REST expression was present in the nuclei and cytoplasm, whereas, in liver tumor tissue, nuclear NRSF/REST staining was clearly reduced and expression was detected in the cytoplasm. Furthermore, the highest levels of NRSF/REST were detected in normal liver tissue $(90 \%$ of cases); however, the expression of NRSF/REST among all liver tumors, including HCC and CCC, did not demonstrate any significant difference compared with the normal liver tissue. However, in CCC tissues, the number of samples with high NRSF/REST expression was significantly decreased compared with normal or HCC tissues, with only $7 \%$ of CCCs exhibiting high levels of NRSF/REST. The expression of NRSF/REST was not statistically different among the grades of tumor differentiation (WDC, MDC and PDC) or between pathological stages. Finally, no age- or sex-associated differences were identified in the number of samples with high NRSF/REST immunoreactivity among all the tissues examined, including normal and cancerous liver tissues.

As a transcription factor, NRSF/REST expression has typically been detected in cell nuclei in previous studies. For example, in human medulloblastoma tumors (13) and normal human brain tissue (26), NRSF/REST staining was observed to be localized to the nuclei. Conti et al (14) also reported that in gliomas, NRSF/REST exhibited a nuclear staining pattern (14). In the present study, positive NRSF/REST staining was detected in the nuclei and cytoplasm in normal liver tissue; whereas, in liver carcinoma, NRSF/REST was predominantly detected in the cytoplasm. Similar results have been previously observed in other tissues. For example, Conti et al (14) also reported that in normal human tissue, NRSF/REST immunoreactivity was detected in the cytoplasm of selected neurons. Furthermore, Orta-Salazar et al (27) reported that in the hippocampus of a 3xTg-AD mouse (a mouse model of Alzheimer's disease), the nuclei and cytoplasm were NRSF/REST-positive. Additionally, these mice exhibited decreased cytoplasmic and increased nuclear NRSF/REST staining compared with control mice, which was indicated to be associated with the degeneration observed in Alzheimer's disease. Lu et al (26) reported that in normal human brain tissue, NRSF/REST was predominantly localized in the cell nuclei; however, in several brain tissue samples from patients with dementia, NRSF/REST was predominantly absent from the cell nuclei, but present in the cytoplasm. This nuclear loss and cytoplasmic translocation was indicated to be one of the causes of the reduced repression of certain dementia/stress-associated genes that are highly expressed in dementia (26). In the present study, nuclear/cytoplasmic translocation of NRSF/REST was clearly observed; this shift indicated that loss of nuclear NRSF/REST may contribute to hepatic carcinogenesis.

It has been previously reported that NRSF/REST may be tumor-suppressive or exert an oncogenic effect (28). Using array-comparative genomic hybridization analysis, Westbrook et al (29) identified that NRSF/REST is frequently deleted in colorectal cancer, and proposed that it functions as a tumor suppressor. Additionally, Blom et al (30) used gene copy number analyses to demonstrate that the majority of brain tumors exhibited low-level amplification of NRSF/REST. Wagoner et al (31) reported that expression of NRSF/REST was lost in aggressive breast cancer, and that this loss was positively correlated with poor prognosis and higher recurrence rates. Similarly, Lv et al (20) reported that NRSF/REST expression was decreased in breast cancer samples. Furthermore, Varghese et al (21) demonstrated that expression of NRSF/REST was reduced in uterine fibroids (leiomyomas). A decreased NRSF/REST expression profile was also detected in human brain tissue from patients with dementia and in the brain tissue from an Alzheimer's disease mouse model $(26,27)$.

However, certain studies have also reported that NRSF/REST expression is increased in individual tumors or other tissues. For example, Lawinger et al (11) reported that NRSF/REST levels were high in three types of human medulloblastoma cells. Furthermore, Fuller et al (13) reported that expression of NRSF/REST was increased in human medulloblastoma tumors compared with normal brain tissue samples (13) and Conti et al (14) observed that NRSF/REST expression was increased in human GBM. The present study demonstrated that the number of samples with high NRSF/REST expression was reduced in CCC compared with normal tissues, indicating that decreased NRSF/REST levels may be associated with the occurrence and/or poor prognosis of CCC.

Liver disease is a major global health concern (32), with liver and intrahepatic bile duct cancer among the top 10 causes of cancer-associated mortality in the United States (25). In China, liver carcinomas (including HCC and CCC) are also commonly diagnosed and have been identified as one of the leading causes of cancer-associated mortality (33). Therefore, it is important to investigate the mechanisms of liver disease and to identify potential novel therapeutic targets. A study by Sedaghat et al (34) reported that in mouse liver, NRSF/REST regulates the expression of neuronal markers, including brain-derived neurotrophic factor and various other genes (a total of 433 genes, of which $25 \%$ were downregulated and $75 \%$ upregulated), particularly those associated with the cell cycle, cell growth, proliferation and cancer.

The present study observed cytoplasmic translocation of NRSF/REST in liver carcinomas compared with NRSF/REST detected in normal liver tissues and the levels of NRSF/REST expression were reduced in CCC. In conclusion, the results of the current and previous studies indicate that NRSF/REST has an important function in liver carcinomas. Furthermore, the observed nuclear/cytoplasmic translocation may contribute to tumor formation and the reduced levels of NRSF/REST 
may potentially be used as a biomarker of CCC. Notably, as NRSF/REST may paradoxically exert tumor suppressive or oncogenic effects (28), the expression and importance of NRSF/REST in normal and abnormal liver tissues requires further investigation.

\section{Acknowledgements}

The present study was supported by the National Science Foundation of China (grant nos. 81571059 and 81270525).

\section{References}

1. Zhao Y, Zhu M, Yu Y, Qiu L, Zhang Y, He L and Zhang J: Brain REST/NRSF is not only a silent repressor but also an active protector. Mol Neurobiol 54: 541-550, 2016.

2. Chong JA, Tapia-Ramírez J, Kim S, Toledo-Aral JJ, Zheng Y, Boutros MC, Altshuller YM, Frohman MA, Kraner SD and Mandel G: REST: A mammalian silencer protein that restricts sodium channel gene expression to neurons. Cell 80: 949-957, 1995.

3. Gupta SK, Gressens P and Mani S: NRSF downregulation induces neuronal differentiation in mouse embryonic stem cells. Differentiation 77: 19-28, 2009.

4. Gao Z, Ure K, Ding P, Nashaat M, Yuan L, Ma J, Hammer RE and Hsieh J: The master negative regulator REST/NRSF controls adult neurogenesis by restraining the neurogenic program in quiescent stem cells. J Neurosci 31: 9772-9786, 2011

5. Chen ZF, Paquette AJ and Anderson DJ: NRSF/REST is required in vivo for repression of multiple neuronal target genes during embryogenesis. Nat Genet 20: 136-142, 1998.

6. Olguín P, Oteíza P, Gamboa E, Gómez-Skármeta JL and Kukuljan M: RE-1 silencer of transcription/neural restrictive silencer factor modulates ectodermal patterning during Xenopus development. J Neurosci 26: 2820-2829, 2006.

7. Paquette AJ, Perez SE and Anderson DJ: Constitutive expression of the neuron-restrictive silencer factor (NRSF)/REST in differentiating neurons disrupts neuronal gene expression and causes axon pathfinding errors in vivo. Proc Natl Acad Sci USA 97: 12318-12323, 2000 .

8. Nishimura E, Sasaki K, Maruyama K, Tsukada T and Yamaguchi K: Decrease in neuron-restrictive silencer factor (NRSF) mRNA levels during differentiation of cultured neuroblastoma cells. Neurosci Lett 211: 101-104, 1996.

9. Lee JH, Chai YG and Hersh LB: Expression patterns of mouse repressor element-1 silencing transcription factor 4 (REST4) and its possible function in neuroblastoma. J Mol Neurosci 15 205-214, 2000

10. Lepagnol-Bestel AM, Maussion G, Ramoz N, Moalic JM, Gorwood P and Simonneau M: Nrsf silencing induces molecular and subcellular changes linked to neuronal plasticity. Neuroreport 18: 441-446, 2007.

11. Lawinger P, Venugopal R, Guo ZS, Immaneni A, Sengupta D, Lu W, Rastelli L, Marin Dias Carneiro A, Levin V,Fuller GN, et al: The neuronal repressor REST/NRSF is an essential regulator in medulloblastoma cells. Nat Med 6: 826-831, 2000.

12. Su X, Gopalakrishnan V, Stearns D, Aldape K, Lang FF, Fuller G, Snyder E, Eberhart CG and Majumder S: Abnormal expression of REST/NRSF and Myc in neural stem/progenitor cells causes cerebellar tumors by blocking neuronal differentiation. Mol Cell Biol 26: 1666-1678, 2006.

13. Fuller GN, Su X, Price RE, Cohen ZR, Lang FF, Sawaya R and Majumder S: Many human medulloblastoma tumors overexpress repressor element-1 silencing transcription (REST)/neuron-restrictive silencer factor, which can be functionally countered by REST-VP16. Mol Cancer Ther 4: 343-349, 2005.

14. Conti L, Crisafulli L, Caldera V, Tortoreto M, Brilli E, Conforti P, Zunino F, Magrassi L, Schiffer D and Cattaneo E: REST controls self-renewal and tumorigenic competence of human glioblastoma cells. PLoS One 7: e38486, 2012.
15. Zhang D, Li Y, Wang R, Li Y, Shi P, Kan Z and Pang X: Inhibition of rest suppresses proliferation and migration in glioblastoma cells. Int J Mol Sci 17: pii: E664, 2016.

16. Gurrola-Diaz C, Lacroix J, Dihlmann S, Becker CM and von Knebel Doeberitz M: Reduced expression of the neuron restrictive silencer factor permits transcription of glycine receptor alpha1 subunit in small-cell lung cancer cells. Oncogene 22: 5636-5645, 2003

17. Watanabe $\mathrm{H}$, Mizutani $\mathrm{T}$, Haraguchi $\mathrm{T}$, Yamamichi $\mathrm{N}$, Minoguchi S, Yamamichi-Nishina M, Mori N, Kameda T, Sugiyama T and Iba H: SWI/SNF complex is essential for NRSF-mediated suppression of neuronal genes in human nonsmall cell lung carcinoma cell lines. Oncogene 25: 470-479, 2006.

18. Kreisler A, Strissel PL, Strick R, Neumann SB, Schumacher U and Becker CM: Regulation of the NRSF/REST gene by methylation and CREB affects the cellular phenotype of small-cell lung cancer. Oncogene 29: 5828-5838, 2010.

19. Bronson MW, Hillenmeyer S, Park RW and Brodsky AS: Estrogen coordinates translation and transcription, revealing a role for NRSF in human breast cancer cells. Mol Endocrinol 24: 1120-1135, 2010.

20. Lv H, Pan G, Zheng G, Wu X, Ren H, Liu Y and Wen J: Expression and functions of the repressor element 1 (RE-1)-silencing transcription factor (REST) in breast cancer. J Cell Biochem 110: 968-974, 2010.

21. Varghese BV, Koohestani F, McWilliams M, Colvin A, Gunewardena S, Kinsey WH, Nowak RA, Nothnick WB and Chennathukuzhi VM: Loss of the repressor REST in uterine fibroids promotes aberrant $\mathrm{G}$ protein-coupled receptor 10 expression and activates mammalian target of rapamycin pathway. Proc Natl Acad Sci USA 110: 2187-2192, 2013.

22. Edge SB and Compton CC: The American Joint Committee on Cancer: The VIIth edition of the AJCC cancer staging manual and the future of TNM. Ann Surg Oncol 17: 1471-1474, 2010.

23. Liu M, Zhang K, Zhao Y, Guo Q, Guo D and Zhang J: Evidence for involvement of steroid receptors and coactivators in neuroepithelial and meningothelial tumors. Tumour Biol 36: 3251-3261, 2015.

24. Liu C, Zhang Y, Zhang K, Bian C, Zhao Y and Zhang J: Expression of estrogen receptors, androgen receptor and steroid receptor coactivator-3 is negatively correlated to the differentiation of astrocytic tumors. Cancer Epidemiol 38: 291-297, 2014.

25. Siegel R, Ma J, Zou Z and Jemal A: Cancer statistics, 2014. CA Cancer J Clin 64: 9-29, 2014.

26. Lu T, Aron L, Zullo J, Pan Y, Kim H, Chen Y, Yang TH, Kim HM, Drake D, Liu XS, et al: REST and stress resistance in ageing and Alzheimer's disease. Nature 507: 448-454, 2014.

27. Orta-Salazar E, Aguilar-Vázquez A, Martínez-Coria $\mathrm{H}$, Luquín-De Anda S, Rivera-Cervantes M, Beas-Zarate C, Feria-Velasco A and Díaz-Cintra S: REST/NRSF-induced changes of ChAT protein expression in the neocortex and hippocampus of the 3xTg-AD mouse model for Alzheimer's disease. Life Sci 116: 83-89, 2014

28. Majumder S: REST in good times and bad: Roles in tumor suppressor and oncogenic activities. Cell Cycle 5: 1929-1935, 2006.

29. Westbrook TF, Martin ES, Schlabach MR, Leng Y, Liang AC, Feng B, Zhao JJ, Roberts TM, Mandel G, Hannon GJ, et al: A genetic screen for candidate tumor suppressors identifies REST. Cell 121: 837-848, 2005

30. Blom T, Tynninen O, Puputti M, Halonen M, Paetau A, Haapasalo H, Tanner M and Nupponen NN: Molecular genetic analysis of the REST/NRSF gene in nervous system tumors. Acta Neuropathol 112: 483-490, 2006

31. Wagoner MP, Gunsalus KT, Schoenike B, Richardson AL, Friedl A and Roopra A: The transcription factor REST is lost in aggressive breast cancer. PLoS Genet 6: e1000979, 2010.

32. Hansel MC, Davila JC, Vosough M, Gramignoli R, Skvorak KJ, Dorko K, Marongiu F, Blake W and Strom SC: The use of induced pluripotent stem cells for the study and treatment of liver diseases. Curr Protoc Toxicol 67: 14.13.1-14.13.27, 2016.

33. Chen W, Zheng R, Baade PD, Zhang S, Zeng H, Bray F, Jemal A, Yu XQ and He J: Cancer statistics in China, 2015. CA Cancer J Clin 66: 115-132, 2016

34. Sedaghat Y, Bui HH, Mazur C and Monia BP: Identification of REST-regulated genes and pathways using a REST-targeted antisense approach. Nucleic Acid Ther 23: 389-400, 2013. 